TRANSACTIONS OF THE

AMERICAN MATHEMATICAL SOCIETY

Volume 364, Number 3, March 2012, Pages 1163-1177

S 0002-9947(2011)05534-0

Article electronically published on November 2, 2011

\title{
SHARP BOUNDS FOR GENERAL COMMUTATORS ON WEIGHTED LEBESGUE SPACES
}

\author{
DAEWON CHUNG, M. CRISTINA PEREYRA, AND CARLOS PEREZ
}

\begin{abstract}
We show that if a linear operator $T$ is bounded on a weighted Lebesgue space $L^{2}(w)$ and obeys a linear bound with respect to the $A_{2}$ constant of the weight, then its commutator $[b, T]$ with a function $b$ in $B M O$ will obey a quadratic bound with respect to the $A_{2}$ constant of the weight. We also prove that the $k$ th-order commutator $T_{b}^{k}=\left[b, T_{b}^{k-1}\right]$ will obey a bound that is a power $(k+1)$ of the $A_{2}$ constant of the weight. Sharp extrapolation provides corresponding $L^{p}(w)$ estimates. In particular these estimates hold for $T$ any Calderón-Zygmund singular integral operator. The results are sharp in terms of the growth of the operator norm with respect to the $A_{p}$ constant of the weight for all $1<p<\infty$, all $k$, and all dimensions, as examples involving the Riesz transforms, power functions and power weights show.
\end{abstract}

\section{INTRODUCTION}

Singular integral operators are known to be bounded in weighted Lebesgue spaces $L^{p}(w)$ if the weight belongs to the $A_{p}$ class of Muckenhoupt.

Recently there has been renewed interest in understanding the dependence of the operator norm in terms of the $A_{p}$ constant of the weight; more precisely, one seeks estimates of the type

$$
\|T\|_{L^{p}(w)} \leq \varphi_{p}\left([w]_{A_{p}}\right) \quad 1<p<\infty,
$$

where the function $\varphi_{p}:[1, \infty) \rightarrow[0, \infty)$ is optimal in terms of growth. The first result of this type was obtained by S. Buckley 4 who showed that the maximal function obeyed such estimates with $\varphi_{p}(t)=c_{p} t^{\frac{1}{p-1}}$ for $1<p<\infty$, and this is optimal (see [21] for another recent proof). This problem has attracted renewed attention because of the work of Astala, Iwaniec and Saksman 2]. They proved sharp regularity results for solutions to the Beltrami equation, assuming that the operator norm of the Beurling-Ahlfors transform grows linearly in terms of the $A_{p}$ constant for $p \geq 2$. This linear growth was proved by S. Petermichl and A. Volberg 32 and by Petermichl 30, 31 for the Hilbert transform and the Riesz transforms. In these papers it has been shown that if $T$ is any of these operators, then

$$
\|T\|_{L^{p}(w)} \leq c_{p, n}[w]_{A_{p}}^{\max \left\{1, \frac{1}{p-1}\right\}} \quad 1<p<\infty,
$$

and the exponent $\max \left\{1, \frac{1}{p-1}\right\}$ is best possible. It has been conjectured, and very recently proved [16, that the same estimate holds for any Calderón-Zygmund

Received by the editors February 11, 2010.

2010 Mathematics Subject Classification. Primary 42B20, 42B25; Secondary 46B70, 47B38.

Key words and phrases. Commutators, singular integrals, $\mathrm{BMO}, A_{2}, A_{p}$.

The third author would like to acknowledge the support of the Spanish Ministry of Science and Innovation via grant MTM2009-08934. 
operator $T$. By the sharp version of the Rubio de Francia extrapolation theorem [12], it suffices to prove this inequality for $p=2$, namely

$$
\|T\|_{L^{2}(w)} \leq c_{n}[w]_{A_{2}} .
$$

We refer the reader to [10 for a new proof and for generalizations and applications. The linear growth in $L^{2}(w)$ has been shown to hold for dyadic operators (martingale transform [34, dyadic square function [15, dyadic paraproduct 3]), or for operators that have lots of symmetries and can be written as averages of dyadic shift operators (such as the Hilbert transform [30, Riesz transforms 31, Beurling transform [32, 13]). All these estimates were obtained using Bellman functions. Recently all the above results have been recovered using different sets of techniques and obtaining linear bounds for the larger class of Haar shift operators [20, 8]. In particular, in the latter paper [8], no Bellman function techniques nor any two weight results are used, and the methods can be extended to other important operators in Harmonic Analysis such as dyadic square functions and paraproducts, maximal singular integrals and the vector-valued maximal function as can be found in 9. The sharp bound (1.2) for any Calderón-Zygmund operator $T$ has been proved in [16 by T. Hytönen. Hytönen's proof is based on approximating $T$ by generalized dyadic Haar shift operators with good bounds combined with the key fact that to prove (1.2) it is enough to prove the corresponding weak type $(2,2)$ estimate with the same linear bound as proved in [29]. A direct proof avoiding this weak $(2,2)$ reduction can be found in [17. A bit earlier, in 22, the sharp $L^{p}(w)$ bound for $T$ was obtained for values of $p$ outside the interval $(3 / 2,3)$ and the proof is based on the corresponding estimates for the intrinsic square functions.

It should be mentioned that until the $A_{2}$-conjecture was proved, only the special case

$$
\|T\|_{L^{p}(w)} \leq C_{p}[w]_{A_{1}}, \quad 1<p<\infty,
$$

had been shown to be true for any Calderón-Zygmund operator. Observe that the condition imposed on the weight is the $A_{1}$ weight condition, which is stronger than $A_{p}$, but there is a gain in the exponent since it is linear for any $1<p<\infty$ (compare with (1.10). This has been shown in [23, 24] and we refer the reader to [28, for a survey on this topic.

The main purpose of this paper is to prove estimates similar to (1.1) for commutators of appropriate linear operators $T$ with $B M O$ functions $b$. These operators are defined formally by the expression

$$
[b, T] f=b T(f)-T(b f) .
$$

When $T$ is a singular integral operator, these operators were considered by Coifman, Rochberg and Weiss in [7. Although the original interest in the study of such operators was related to generalizations of the classical factorization theorem for Hardy spaces, many other applications have been found.

The main result from 7 states that $[b, T]$ is a bounded operator on $L^{p}\left(\mathbb{R}^{n}\right)$, $1<p<\infty$, when $b$ is a $B M O$ function and $T$ is a singular integral operator. In fact, the $B M O$ condition of $b$ is also a necessary condition for the $L^{p}$-boundedness of the commutator when $T$ is the Hilbert transform. Later on a different proof was given by J. O. Strömberg (cf. [33, p. 417) with the advantage that it allows us to show that these commutators are also bounded on weighted $L^{p}(w)$, when $w \in A_{p}$. This approach is based on the use of the classical C. Fefferman-Stein maximal 
function and it is not precise enough for further developments. Indeed, we may think that these operators behave as Calderón-Zygmund operators; however, there are some differences. For instance, an interesting fact is that, unlike what is done with singular integral operators, the proof of the $L^{p}$-boundedness of the commutator does not rely on a weak type $(1,1)$ inequality. In fact, simple examples show that in general $[b, T]$ fails to be of weak type $(1,1)$ when $b \in B M O$. This was observed by the third author in [26], where it is also shown that there is an appropriate weak- $L(\log L)$ type estimate replacement. This shows that the operator cannot be a Calderón-Zygmund singular integral operator. To stress this point of view it is also shown by the third author [27] that the right operator controlling $[b, T]$ is $M^{2}=M \circ M$, instead of the Hardy-Littlewood maximal function $M$.

In the present paper we pursue this point of view by showing that commutators have an extra "bad" behavior from the point of view of the $A_{p}$ theory of weights that is not reflected in the classical situation. Our argument will be based on the second proof for the $L^{p}$-boundedness of the commutator presented in [7]. This proof is interesting because there is no need to assume that $T$ is a singular integral operator; to show the boundedness of the commutator it is enough to assume that the operator $T$ is linear and bounded on $L^{p}(w)$ for any $w \in A_{p}$. These ideas were exploited in 1 .

The first author showed in 5 that the commutator with the Hilbert transform obeys an estimate of the following type,

$$
\|[b, H]\|_{L^{2}(w)} \leq C[w]_{A_{2}}^{2}\|b\|_{B M O},
$$

and he also showed that the quadratic growth with respect to the $A_{2}$ constant of the weight is sharp. The techniques used in that paper rely very much on dyadic considerations and the use of Bellman function arguments. Using recent results on Haar shift operators, he also deduced the quadratic growth for commutators of Haar shift operators and operators in their convex hull, including the Riesz transforms and the Beurling-Ahlfors operator; see 5 for the details.

Also, it should be mentioned that there is a corresponding version of (1.3) for commutators of any Calderón-Zygmund operator with quadratic growth as in (1.4). This is proved in 25] where an endpoint estimate can also be found.

By completely different methods, we show in this paper that if an operator obeys an initial linear bound in $L^{2}(w)$, then its commutator will obey a quadratic bound in $L^{2}(w)$. In light of the positive resolution of the $A_{2}$-conjecture, we conclude that the commutator of any Calderón-Zygmund singular integral operator and a $B M O$ function obeys a quadratic bound in $L^{2}(w)$. In fact we show that if an operator $T$ obeys a bound in $L^{2}(w)$ of the form $\varphi\left([w]_{A_{2}}\right)$, then its $k$ th order commutator with $b \in B M O, T_{b}^{k}:=\left[b, T_{b}^{k-1}\right]$, will obey a bound of the form $c_{n}^{k} k ! \varphi\left(\gamma_{n}[w]_{A_{2}}\right)[w]_{A_{2}}^{k}\|b\|_{B M O}^{k}$. Observe that if we consider the special case of Calderón-Zygmund operators with kernel $K$, then

$$
T_{b}^{k}(f)(x) \int_{\mathbb{R}^{n}}(b(x)-b(y))^{k} K(x, y) f(y) d y,
$$

and the larger $k$ is, the more singular the operator will be, because the exponent in $[w]_{A_{2}}^{k}$ becomes larger.

Corresponding estimates in $L^{p}(w)$ are deduced by the sharp version of the Rubio de Francia extrapolation theorem found in [12, and are shown to be sharp in the 
case of the Hilbert and Riesz transforms (in any dimension) for all $1<p<\infty$, and for all $k \geq 1$.

It will be interesting to recover the result for the higher-order commutators with the Haar shift operators (and hence for the Hilbert, Riesz and Beurling transforms) using the dyadic methods, but so far we do not know how to do this.

Recently extensions of our result to two weight settings, fractional integrals and more have been obtained by D. Cruz-Uribe and Kabe Moen; see 11.

The remainder of this paper is organized as follows. In Section 2 we gather some basic results. In Section 3 we give the proof of the main result. In Section 4 we show with examples that the main theorem in the paper and its corollaries are sharp. Finally, the last section is an appendix where we show a result that is claimed but never proved in the literature, a sharp reverse Hölder's inequality for $A_{2}$ weights.

\section{Preliminary Results}

2.1. A sharp John-Nirenberg. For a locally integrable $b: \mathbb{R}^{n} \rightarrow \mathbb{R}$ we define

$$
\|b\|_{B M O}=\sup _{Q} \frac{1}{|Q|} \int_{Q}\left|b(y)-b_{Q}\right| d y<\infty
$$

where the supremum is taken over all cubes $Q \in \mathbb{R}^{n}$ with sides parallel to the axes, and

$$
b_{Q}=\frac{1}{|Q|} \int_{Q} b(y) d y
$$

The main relevance of $B M O$ is because of its exponential self-improving property, recorded in the celebrated John-Nirenberg Theorem 18. We need a very precise version of it, as follows:

Theorem 2.1 (Sharp John-Nirenberg). There are dimensional constants $0 \leq \alpha_{n}<$ $1<\beta_{n}$ such that

$$
\sup _{Q} \frac{1}{|Q|} \int_{Q} \exp \left(\frac{\alpha_{n}}{\|b\|_{B M O}}\left|b(y)-b_{Q}\right|\right) d y \leq \beta_{n} .
$$

In fact we can take $\alpha_{n}=\frac{1}{2^{n+2}}$.

For the proof of this we refer to pp. 31-32 of [19], where a proof different from the standard one can be found.

We derive from Theorem 2.1 the following Lemma 2.2 which will be used in the proof of the main theorem. First recall that a weight $w$ satisfies the $A_{2}$ condition if

$$
[w]_{A_{2}}=\sup _{Q}\left(\frac{1}{|Q|} \int_{Q} w\right)\left(\frac{1}{|Q|} \int_{Q} w^{-1}\right)<\infty,
$$

where the supremum is taken over all cubes $Q \in \mathbb{R}^{n}$ with sides parallel to the axes. Notice that $[w]_{A_{2}} \geq 1$.

It is well known that if $w \in A_{2}$, then $b=\log w \in B M O$. A partial converse also holds: if $b \in B M O$, there is an $s_{0}>0$ such that $w=e^{s b} \in A_{p},|s| \leq s_{0}$. As a consequence of the Sharp John-Nirenberg Theorem we can obtain a more precise version of this partial converse.

Lemma 2.2. Let $b \in B M O$ and let $\alpha_{n}<1<\beta_{n}$ be the dimensional constants from (2.1). Then

$$
s \in \mathbb{R}, \quad|s| \leq \frac{\alpha_{n}}{\|b\|_{B M O}} \Longrightarrow e^{s b} \in A_{2} \quad \text { and } \quad\left[e^{s b}\right]_{A_{2}} \leq \beta_{n}^{2} .
$$


Proof. By Theorem 2.1, if $|s| \leq \frac{\alpha_{n}}{\|b\|_{B M O}}$ and if $Q$ is fixed,

$$
\frac{1}{|Q|} \int_{Q} \exp \left(|s|\left|b(y)-b_{Q}\right|\right) d y \leq \frac{1}{|Q|} \int_{Q} \exp \left(\frac{\alpha_{n}}{\|b\|_{B M O}}\left|b(y)-b_{Q}\right|\right) d y \leq \beta_{n} ;
$$

thus

$$
\frac{1}{|Q|} \int_{Q} \exp \left(s\left(b(y)-b_{Q}\right)\right) d y \leq \beta_{n}
$$

and

$$
\frac{1}{|Q|} \int_{Q} \exp \left(-s\left(b(y)-b_{Q}\right)\right) d y \leq \beta_{n} .
$$

If we multiply the inequalities, the $b_{Q}$ parts cancel out

$$
\begin{gathered}
\left(\frac{1}{|Q|} \int_{Q} \exp \left(s\left(b(y)-b_{Q}\right)\right) d y\right)\left(\frac{1}{|Q|} \int_{Q} \exp \left(s\left(b_{Q}-b(y)\right)\right) d y\right) \\
=\left(\frac{1}{|Q|} \int_{Q} \exp (s b(y)) d y\right)\left(\frac{1}{|Q|} \int_{Q} \exp (-s b(y)) d y\right) \leq \beta_{n}^{2}
\end{gathered}
$$

namely, $e^{s b} \in A_{2}$ with

$$
\left[e^{s b}\right]_{A_{2}} \leq \beta_{n}^{2}
$$

We remark that it follows easily from minor modifications to the proof of Lemma 2.2 that if $1<p<\infty$,

$$
s \in \mathbb{R}, \quad|s| \leq \frac{\alpha_{n}}{\|b\|_{B M O}} \min \left\{1, \frac{1}{p-1}\right\} \Longrightarrow e^{s b} \in A_{p} \quad \text { and } \quad\left[e^{s b}\right]_{A_{p}} \leq \beta_{n}^{p},
$$

where as usual

$$
[w]_{A_{p}}=\sup _{Q}\left(\frac{1}{|Q|} \int_{Q} w(x) d x\right)\left(\frac{1}{|Q|} \int_{Q} w(x)^{-1 /(p-1)} d x\right)^{p-1}<\infty .
$$

2.2. Sharp reverse Hölder inequality for the $A_{2}$ class of weights. Recall that if $w \in A_{2}$, then $w$ satisfies a reverse Hölder condition; namely, there are constants $r>1$ and $c \geq 1$ such that for any cube $Q$,

$$
\left(\frac{1}{|Q|} \int_{Q} w^{r} d x\right)^{\frac{1}{r}} \leq \frac{c}{|Q|} \int_{Q} w .
$$

In the usual proofs, both constants, $c$ and $r$, depend upon the $A_{2}$ constant of the weight. There is a more precise version of (2.2).

Lemma 2.3. Let $w \in A_{2}$ and let $r_{w}=1+\frac{1}{2^{n+5}[w]_{A_{2}}}$. Then

$$
\left(\frac{1}{|Q|} \int_{Q} w^{r_{w}} d x\right)^{\frac{1}{r_{w}}} \leq \frac{2}{|Q|} \int_{Q} w .
$$

This result was stated and used in [4 but no proof was given. The author mentioned instead the celebrated work [6] where no explicit statement can be found. We supply in Section 5 a proof taken from [28, where a more general version can be found as well as more information. 


\section{Main Result}

Theorem 3.1. Let $T$ be a linear operator bounded on $L^{2}(w)$ for any $w \in A_{2}$. Suppose further that there is an increasing function $\varphi:[1, \infty) \rightarrow[0, \infty)$ such that

$$
\|T\|_{L^{2}(w)} \leq \varphi\left([w]_{A_{2}}\right) .
$$

Then there are constants $\gamma_{n}$ and $c_{n}$ independent of $[w]_{A_{2}}$ such that

$$
\|[b, T]\|_{L^{2}(w)} \leq c_{n} \varphi\left(\gamma_{n}[w]_{A_{2}}\right)[w]_{A_{2}}\|b\|_{B M O} .
$$

For the particular case $\varphi(t)=c_{0} t^{r}$, where $r>0$, and $c_{0}>0$, a simple induction argument shows that if

$$
\|T\|_{L^{2}(w)} \leq a_{0}[w]_{A_{2}}^{r}
$$

then for each integer $k \geq 1$ there is a constant $a_{k}$ depending on $k$, the initial value $a_{0}$, and the parameters $\gamma_{n}$ and $c_{n}$ in the theorem, such that the $k$ th-order commutator $T_{b}^{k}$, defined recursively by $T_{b}^{k}:=\left[b, T_{b}^{k-1}\right]$, obeys the following weighted estimates:

$$
\left\|T_{b}^{k}\right\|_{L^{2}(w)} \leq a_{k}[w]_{A_{2}}^{r+k}\|b\|_{B M O}^{k} .
$$

More precisely, the sequence $\left\{a_{k}\right\}_{k \geq 0}$ obeys the following recurrence equation that can be solved easily:

$$
a_{k}=c_{n} a_{k-1} \gamma_{n}^{r+k-1}=c_{n}^{k} a_{0} \gamma_{n}^{k r+\frac{(k-2)(k-1)}{2}} .
$$

Using the method of proof of Theorem 3.1 we can obtain a weighted estimate for the $k$ th-order commutator that works for a general increasing function $\varphi:[1, \infty) \rightarrow$ $[0, \infty)$. Notice the difference in the constants with what we just argued by induction for the particular case $\varphi(t)=a_{0} t^{r}$ : in the corollary the constant is $c_{n}^{k} a_{0} \gamma_{n}^{r} k !$, whereas in the induction argument the constant is $c_{n}^{k} a_{0} \gamma_{n}^{k r+\frac{(k-2)(k-1)}{2}}$.

Corollary 3.2. Let $T$ be a bounded linear operator on $L^{2}(w)$ with $w \in A_{2}$ and

$$
\|T\|_{L^{2}(w)} \leq \varphi\left([w]_{A_{2}}\right)
$$

Then there are constants $\gamma_{n}$ and $c_{n}$ independent of $[w]_{A_{2}}$ such that

$$
\left\|T_{b}^{k}\right\|_{L^{2}(w)} \leq c_{n}^{k} k ! \varphi\left(\gamma_{n}[w]_{A_{2}}\right)[w]_{A_{2}}^{k}\|b\|_{B M O}^{k} .
$$

The constants $\gamma_{n}$ and $c_{n}$ that appear in Corollary 3.2 are the same as those that appeared in Theorem [3.1. We first present the proof of Theorem 3.1] and afterwards we discuss the necessary modifications to obtain Corollary 3.2 As an easy consequence of Corollary 3.2 and the Rubio de Francia extrapolation theorem with sharp constants [12], we have the following.

Corollary 3.3. Let $T$ be a linear operator bounded on $L^{2}(w)$ with $w \in A_{2}$ and

$$
\|T\|_{L^{2}(w)} \leq \varphi\left([w]_{A_{2}}\right) .
$$

Then, for $1<p<\infty$, there are constants $\gamma_{n, p}$ and $c_{n, p}$, which only depend on $p$, and the dimension $n$, such that for all weights $w \in A_{p}$,

$$
\left\|T_{b}^{k}\right\|_{L^{p}(w)} \leq \sqrt{2} c_{n, p}^{k} k ! \varphi\left(\gamma_{n, p}[w]_{A_{p}}^{\max \left\{1, \frac{1}{p-1}\right\}}\right)[w]_{A_{p}}^{k \max \left\{1, \frac{1}{p-1}\right\}}\|b\|_{B M O}^{k} .
$$


In the particular case $\varphi(t)=a_{0} t^{r}$, the extrapolated estimate looks like

$$
\left\|T_{b}^{k}\right\|_{L^{p}(w)} \leq \sqrt{2} a_{0} c_{n}^{k} k ! \gamma_{n}^{r} c_{p}^{r+k}[w]_{A_{p}}^{(r+k) \max \left\{1, \frac{1}{p-1}\right\}}\|b\|_{B M O}^{k},
$$

where $c_{p}$ depends only on $p$, and where $\gamma_{n}$ and $c_{n}$ are the constants that appeared in Theorem 3.1

We will show in Section 4 that for $r=1, \varphi(t)=a_{0} t$, the power $(1+k) \max \left\{1, \frac{1}{p-1}\right\}$ cannot be decreased for $T=H$ and $T=R_{j}$ the Hilbert and Riesz transforms, for all $k \geq 1$ and $p>1$; therefore the theorem is optimal in terms of the rate of the dependence on $[w]_{A_{p}}$. In [5], examples for $k=1$, for all $p>1$, and for $T$ the Hilbert, Beurling and Riesz transforms, were presented.

Proof of Theorem 3.1. We "conjugate" the operator as follows: if $z$ is any complex number we define

$$
T_{z}(f)=e^{z b} T\left(e^{-z b} f\right) .
$$

Then, a computation gives (for instance for "nice" functions),

$$
[b, T](f)=\left.\frac{d}{d z} T_{z}(f)\right|_{z=0}=\frac{1}{2 \pi i} \int_{|z|=\epsilon} \frac{T_{z}(f)}{z^{2}} d z, \quad \epsilon>0
$$

by the Cauchy integral theorem; see [7, [1].

Now, by Minkowski's inequality,

$$
\|[b, T](f)\|_{L^{2}(w)} \leq \frac{1}{2 \pi \epsilon^{2}} \int_{|z|=\epsilon}\left\|T_{z}(f)\right\|_{L^{2}(w)}|d z|, \quad \epsilon>0 .
$$

The key point is to find the appropriate radius $\epsilon$. To do this we look at the inner norm $\left\|T_{z}(f)\right\|_{L^{2}(w)}$,

$$
\left\|T_{z}(f)\right\|_{L^{2}(w)}=\left\|T\left(e^{-z b} f\right)\right\|_{L^{2}\left(w e^{2 R e z b}\right)},
$$

and try to find appropriate bounds on $z$. To do this we use the main hypothesis; namely, that $T$ is bounded on $L^{2}(w)$ if $w \in A_{2}$ with

$$
\|T\|_{L^{2}(w)} \leq \varphi\left([w]_{A_{2}}\right)
$$

Hence we should compute

$$
\left[w e^{2 \operatorname{Re} z b}\right]_{A_{2}}=\sup _{Q}\left(\frac{1}{|Q|} \int_{Q} w e^{2 \operatorname{Re} z b(x)} d x\right)\left(\frac{1}{|Q|} \int_{Q} w^{-1} e^{-2 \operatorname{Re} z b(x)} d x\right) .
$$

Now, since $w \in A_{2}$ we use Lemma 2.3, if $r=r_{w}=1+\frac{1}{2^{n+5}[w]_{A_{2}}}<2$, then

$$
\left(\frac{1}{|Q|} \int_{Q} w^{r} d x\right)^{\frac{1}{r}} \leq \frac{2}{|Q|} \int_{Q} w
$$

and similarly for $w^{-1}$ since $r_{w}=r_{w^{-1}}$,

$$
\left(\frac{1}{|Q|} \int_{Q} w^{-r} d x\right)^{\frac{1}{r}} \leq \frac{2}{|Q|} \int_{Q} w^{-1} .
$$


Using this and Hölder's inequality we have for an arbitrary $Q$,

$$
\begin{aligned}
\left(\frac{1}{|Q|} \int_{Q} w(x) e^{2 \operatorname{Re} z b(x)} d x\right)\left(\frac{1}{|Q|} \int_{Q} w(x)^{-1} e^{-2 \operatorname{Re} z b(x)} d x\right) \\
\leq\left(\frac{1}{|Q|} \int_{Q} w^{r} d x\right)^{\frac{1}{r}}\left(\frac{1}{|Q|} \int_{Q} e^{2 \operatorname{Re} z r^{\prime} b(x)} d x\right)^{\frac{1}{r^{\prime}}} \\
\quad \times\left(\frac{1}{|Q|} \int_{Q} w^{-r} d x\right)^{\frac{1}{r}}\left(\frac{1}{|Q|} \int_{Q} e^{-2 \operatorname{Re} z r^{\prime} b(x)} d x\right)^{\frac{1}{r^{\prime}}} \\
\leq 4\left(\frac{1}{|Q|} \int_{Q} w d x\right)\left(\frac{1}{|Q|} \int_{Q} w^{-1} d x\right) \\
\quad \times\left(\frac{1}{|Q|} \int_{Q} e^{2 \operatorname{Re} z r^{\prime} b(x)} d x\right)^{\frac{1}{r^{\prime}}}\left(\frac{1}{|Q|} \int_{Q} e^{-2 \operatorname{Re} z r^{\prime} b(x)} d x\right)^{\frac{1}{r^{\prime}}} \\
\leq 4[w]_{A_{2}}\left[e^{\left.2 \operatorname{Re} z r^{\prime} b\right]_{A_{2}}^{\frac{1}{r^{\prime}}} .}\right.
\end{aligned}
$$

Now, since $b \in B M O$ we are in a position to apply Lemma 2.2

$$
\text { if } \quad\left|2 \operatorname{Re} z r^{\prime}\right| \leq \frac{\alpha_{n}}{\|b\|_{B M O}}, \quad \text { then } \quad\left[e^{2 \operatorname{Re} z r^{\prime} b}\right]_{A_{2}} \leq \beta_{n}^{2} .
$$

Hence for these $z$, and since $1<r<2$,

$$
\left[w e^{2 \operatorname{Re} z b}\right]_{A_{2}} \leq 4[w]_{A_{2}} \beta_{n}^{\frac{2}{r^{\prime}}} \leq 4[w]_{A_{2}} \beta_{n} .
$$

Using this estimate for these $z$, and observing that $\left\|e^{-z b} f\right\|_{L^{2}\left(w e^{2 \operatorname{Re} z b}\right)}=\|f\|_{L^{2}(w)}$,

$$
\begin{aligned}
\left\|T_{z}(f)\right\|_{L^{2}(w)} & =\left\|T\left(e^{-z b} f\right)\right\|_{L^{2}\left(w e^{2 \operatorname{Re} z b}\right)} \\
& \leq \varphi\left(\left[w e^{2 \operatorname{Re} z b}\right]_{A_{2}}\right)\|f\|_{L^{2}(w)} \leq \varphi\left(4[w]_{A_{2}} \beta_{n}\right)\|f\|_{L^{2}(w)} .
\end{aligned}
$$

Now choosing the radius

$$
\epsilon=\frac{\alpha_{n}}{2 r^{\prime}\|b\|_{B M O}}
$$

we can continue estimating the norm in (3.8):

$$
\begin{gathered}
\|[b, T](f)\|_{L^{2}(w)} \leq \frac{1}{2 \pi \epsilon^{2}} \int_{|z|=\epsilon}\left\|T_{z}(f)\right\|_{L^{2}(w)}|d z| \\
\leq \frac{1}{2 \pi \epsilon^{2}} \int_{|z|=\epsilon} \varphi\left(4[w]_{A_{2}} \beta_{n}\right)\|f\|_{L^{2}(w)}|d z|=\frac{1}{\epsilon} \varphi\left(4[w]_{A_{2}} \beta_{n}\right)\|f\|_{L^{2}(w)},
\end{gathered}
$$

since

$$
\left|2 \operatorname{Re} z r^{\prime}\right| \leq 2|z| r^{\prime}=2 \epsilon r^{\prime}=\frac{\alpha_{n}}{\|b\|_{B M O}} .
$$

Finally, for this $\epsilon$,

$$
\|[b, T](f)\|_{L^{2}(w)} \leq C 2^{2 n} \varphi\left(4[w]_{A_{2}} \beta_{n}\right)[w]_{A_{2}}\|b\|_{B M O},
$$

because $r^{\prime}=1+2^{n+5}[w]_{2} \approx 2^{n}[w]_{2}$, and $\alpha_{n}=\frac{1}{2^{n+2}}$.

Observe that the optimal radius is essentially the inverse of $[w]_{2}\|b\|_{B M O}$. This proves the theorem with $c_{n} \sim 2^{2 n}$ and $\gamma_{n}=4 \beta_{n}$. 
Proof of Corollary 3.2. In this case a computation gives (for instance for "nice" functions), see [1] for example or the original paper [7,

$$
T_{b}^{k}(f)=\left.\frac{d^{k}}{d z^{k}} T_{z}(f)\right|_{z=0}=\frac{k !}{2 \pi i} \int_{|z|=\epsilon} \frac{T_{z}(f)}{z^{k+1}} d z, \quad \epsilon>0
$$

by the Cauchy integral theorem. The same calculation as in the case $k=1$ gives the required estimate, with $c_{n}^{k} \approx 2^{2 n k}$, and the same $\gamma_{n}=4 \beta_{n}$.

\section{EXAMPLES}

In this section, we show that one cannot have estimates better than Theorem 3.1. Corollary 3.2 and Corollary 3.3. We present examples which return the same growth with respect to the $A_{p}$ constant of the weight that appears in our results. First, we discuss the simpler case in dimension one. The following example shows that the quadratic estimate for the first commutator of the Hilbert transform is sharp for $p=2$.

4.1. Sharp example for the commutator of the Hilbert transform. Consider the Hilbert transform

$$
H f(x)=p \cdot v \cdot \int_{\mathbb{R}} \frac{f(y)}{x-y} d y,
$$

and consider the $B M O$ function $b(x)=\log |x|$. We know that there is a constant $c$ such that

$$
\|[b, H]\|_{L^{2}(w)} \leq c[w]_{A_{2}}^{2}
$$

and we show that the result is sharp. More precisely, for any increasing function $\phi:[1, \infty) \rightarrow[0, \infty)$ such that $\lim _{t \rightarrow \infty} \frac{t^{2}}{\phi(t)}=\infty$, then

$$
\sup _{w \in A_{2}} \frac{1}{\phi\left([w]_{2}\right)}\|[b, T]\|_{L^{2}(w)}=\infty .
$$

In particular if $\phi(t)=t^{2-\epsilon}$ for any $\epsilon>0$, then (4.2) must hold.

For $0<\delta<1$, we let $w(x)=|x|^{1-\delta}$ and it is easy to see that $[w]_{A_{2}} \sim 1 / \delta$. We now consider the function

$$
f(x)=x^{-1+\delta} \chi_{(0,1)}(x)
$$

and observe that $f$ is in $L^{2}(w)$ with $\|f\|_{L^{2}(w)}=1 / \sqrt{\delta}$. To estimate the $L^{2}(w)$-norm of $[b, H] f$, we claim that

$$
|[b, H] f(x)| \geq \frac{1}{\delta^{2}} f(x)
$$

and hence

$$
\|[b, H] f\|_{L^{2}(w)} \geq \frac{1}{\delta^{2}}\|f\|_{L^{2}(w)},
$$

from which the sharpness (4.2) will follow.

We now prove the claim: if $0<x<1$,

$$
\begin{aligned}
{[b, H] f(x) } & =\int_{0}^{1} \frac{\log (x)-\log (y)}{x-y} y^{-1+\delta} d y=\int_{0}^{1} \frac{\log \left(\frac{x}{y}\right)}{x-y} y^{-1+\delta} d y \\
& =x^{-1+\delta} \int_{0}^{1 / x} \frac{\log \left(\frac{1}{t}\right)}{1-t} t^{-1+\delta} d t .
\end{aligned}
$$


Now,

$$
\int_{0}^{1 / x} \frac{\log \left(\frac{1}{t}\right)}{1-t} t^{-1+\delta} d t=\int_{0}^{1} \frac{\log \left(\frac{1}{t}\right)}{1-t} t^{-1+\delta} d t+\int_{1}^{1 / x} \frac{\log \left(\frac{1}{t}\right)}{1-t} t^{-1+\delta} d t
$$

and since $\frac{\log \left(\frac{1}{t}\right)}{1-t}$ is positive for $(0,1) \cup(1, \infty)$ we have for $0<x<1$,

$$
|[b, H] f(x)|>x^{-1+\delta} \int_{0}^{1} \frac{\log \left(\frac{1}{t}\right)}{1-t} t^{-1+\delta} d t .
$$

But since

$$
\int_{0}^{1} \frac{\log \left(\frac{1}{t}\right)}{1-t} t^{-1+\delta} d t>\int_{0}^{1} \log \left(\frac{1}{t}\right) t^{-1+\delta} d t=\int_{0}^{\infty} s e^{-s \delta} d s=\frac{1}{\delta^{2}},
$$

the claim

$$
|[b, H] f(x)| \geq \frac{1}{\delta^{2}} f(x)
$$

follows. One can find this example and similar examples which show the commutators with the Beurling-Ahlfors operator, and the Riesz transforms obey the quadratic growth in [5].

4.2. Sharp example for the $k$ th-order commutator with the Riesz transforms. We now consider the higher-order commutators with degree $k \geq 1$ in the case of the $j$ th directional Riesz transform on $\mathbb{R}^{n}$ :

$$
R_{j, b}^{k} f(x):=p . v \cdot \int_{\mathbb{R}^{n}} \frac{\left(x_{j}-y_{j}\right)(b(x)-b(y))^{k} f(y)}{|x-y|^{n+1}} d y .
$$

To demonstrate the sharpness of the estimate for the higher-order commutator with the Riesz transforms, we show sharpness for $1<p \leq 2$. Then we can extend the sharpness for all $1<p<\infty$ by using a duality argument, because $R_{j}^{*}=-R_{j}$, so the higher-order commutators of the Riesz transforms are almost selfadjoint operators. For $1<p \leq 2$, we consider weights $\left.w(x)=|x|^{(n-\delta)(p-1)}, f(x)\right] !=|x|^{\delta-n} \chi_{E}(x)$, where $E=\left\{y \mid y \in(0,1)^{n} \cap B(0,1)\right\}, b(x)=\log |x|$, and evaluate the $L^{p}(w)$-norm over $\Omega=\left\{x \in B(0,1)^{c} \mid x_{i}<0\right.$ for all $\left.i=1,2, \ldots, n\right\}$. Note that, for all $y \in E$ and $x \in \Omega$,

$$
\left|x_{j}-y_{j}\right| \geq\left|x_{j}\right| \text { and }|x-y| \leq|y|+|x| .
$$

Then for $x \in \Omega$,

$$
\begin{aligned}
\left|R_{j, b}^{k} f(x)\right| & =\left|\int_{E} \frac{\left(x_{j}-y_{j}\right)(\log |x|-\log |y|)^{k}|y|^{\delta-n}}{|x-y|^{n+1}} d y\right| \\
& =\int_{E} \frac{\left|x_{j}-y_{j}\right|(\log (|x| /|y|))^{k}|y|^{\delta-n}}{|x-y|^{n+1}} d y \geq\left|x_{j}\right| \int_{E} \frac{(\log (|x| /|y|))^{k}|y|^{\delta-n}}{(|y|+|x|)^{n+1}} d y \\
& =\left|x_{j}\right| \int_{E \cap S^{n-1}} \int_{0}^{1} \frac{(\log (|x| / r))^{k} r^{\delta-n} r^{n-1}}{(r+|x|)^{n+1}} d r d \sigma \\
& =c\left|x_{j}\right| \int_{0}^{1 /|x|} \frac{(\log (1 / t))^{k}(t|x|)^{\delta-1}|x|}{(|x|+t|x|)^{n+1}} d t \\
& =\frac{c\left|x_{j}\right|}{|x|^{n+1-\delta}} \int_{0}^{1 /|x|} \frac{(\log (1 / t))^{k} t^{\delta-1}}{(1+t)^{n+1}} d t \\
& \geq \frac{c\left|x_{j}\right|}{|x|^{n+1-\delta}}\left(\frac{|x|}{|x|+1}\right)^{n+1} \int_{0}^{1 /|x|}(\log (1 / t))^{k} t^{\delta-1} d t
\end{aligned}
$$


Note that the constant $c=c(n)$ is the surface measure of $E \cap S^{n-1}$ and depends on the dimension only. On the other hand,

$$
\begin{aligned}
\int_{0}^{1 /|x|}(\log (1 / t))^{k} t^{\delta-1} d t & =\int_{\log |x|}^{\infty} s^{k} e^{-\delta s} d s=-\left.\frac{1}{\delta} e^{-\delta s} s^{k}\right|_{\log |x|} ^{\infty}+\frac{k}{\delta} \int_{\log |x|}^{\infty} e^{-\delta s} s^{k-1} d s \\
& =\frac{1}{\delta} e^{-\delta \log |x|}(\log |x|)^{k}+\frac{k}{\delta} \int_{\log |x|}^{\infty} e^{-\delta s} s^{k-1} d s \\
& =\frac{(\log |x|)^{k}}{\delta|x|^{\delta}}+\frac{k}{\delta} \int_{\log |x|}^{\infty} e^{-\delta s} s^{k-1} d s .
\end{aligned}
$$

For $x \in \Omega,(\log |x|)^{k} /\left(\delta|x|^{\delta}\right)$ is positive; therefore, after neglecting some positive terms and applying the integration by parts $k-1$ times, we get

$$
\begin{aligned}
\int_{0}^{1 /|x|}(\log (1 / t))^{k} t^{\delta-1} d t & \geq \frac{k}{\delta} \int_{\log |x|}^{\infty} e^{-\delta s} s^{k-1} d s \\
& \geq \cdots \geq \frac{k !}{\delta^{k-1}} \int_{\log |x|}^{\infty} e^{-\delta s} s d s \geq \frac{k !}{\delta^{k+1}|x|^{\delta}} .
\end{aligned}
$$

Combining the previous computations, we have that for $x \in \Omega$, and recalling that $f(x)=|x|^{\delta-n} \chi_{E}(x)$,

Thus, we estimate

$$
\left|R_{j, b}^{k} f(x)\right| \geq \frac{k ! c\left|x_{j}\right|}{\delta^{k+1}(|x|+1)^{n+1}} .
$$

$$
\begin{aligned}
\left\|R_{j, b}^{k} f\right\|_{L^{p}(w)}^{p} & \geq \int_{\Omega}\left(\frac{k ! c\left|x_{j}\right|}{\delta^{k+1}(|x|+1)^{n+1}}\right)^{p}|x|^{(n-\delta)(p-1)} d x \\
& =\left(\frac{k ! c}{\delta^{k+1}}\right)^{p} \int_{\Omega} \frac{\left|x_{j}\right|^{p}|x|^{(n-\delta)(p-1)}}{(|x|+1)^{p(n+1)}} d x \\
& \geq\left(\frac{k ! c}{\delta^{k+1}}\right)^{p} \int_{\Omega \cap S^{n-1}} \int_{1}^{\infty} \frac{\gamma_{j}^{p} r^{p} r^{(n-\delta)(p-1)} r^{n-1}}{(r+1)^{p(n+1)}} d r d \sigma(\gamma) \\
& =\left(\frac{k ! c_{n, p}}{\delta^{k+1}}\right)^{p} \int_{1}^{\infty} r^{\delta(1-p)-1} d r=(k !)^{p} \frac{c_{n, p}^{p}}{p-1} \delta^{-p(k+1)-1} .
\end{aligned}
$$

Since $\|f\|_{L^{p}(w)}^{p}=1 / \delta$, and $[w]_{A_{p}} \sim 1 / \delta^{p-1}$, we conclude that

$$
\left\|R_{j, b}^{k} f\right\|_{L^{p}(w)} \geq k ! \frac{c_{n, p}}{(p-1)^{1 / p}} \delta^{-(k+1)-1 / p} \sim k ![w]_{A_{p}}^{\frac{k+1}{p-1}}\|f\|_{L^{p}(w)} .
$$

This shows that Theorem 3.1. Corollary 3.2, and Corollary 3.3 are sharp in the multidimensional case. The constant $c_{n, p} \rightarrow c_{n, 1}>0$ as $p \rightarrow 1$; therefore the estimate blows up as $p \rightarrow 1$, as it should since the operators are not bounded in $L^{1}(w)$.

\section{Appendix: The sharp Reverse Hölder's inequality for $A_{2}$ Weights}

In this section we give a proof of Lemma 2.3 namely, if $w \in A_{2}$, then

$$
\left(\frac{1}{|Q|} \int_{Q} w^{r_{w}} d x\right)^{\frac{1}{r_{w}}} \leq \frac{2}{|Q|} \int_{Q} w
$$

where $r_{w}=1+\frac{1}{2^{5+n}[w]_{A_{2}}}$. 
Proof. Let $w_{Q}=\frac{1}{|Q|} \int_{Q} w$ and $\delta>0$. Then

$$
\begin{aligned}
& \frac{1}{|Q|} \int_{Q} w(x)^{1+\delta} d x=\frac{1}{|Q|} \int_{Q} w(x)^{\delta} w(x) d x=\frac{\delta}{|Q|} \int_{0}^{\infty} \lambda^{\delta} w(\{x \in Q: w(x)>\lambda\}) \frac{d \lambda}{\lambda} \\
& =\frac{\delta}{|Q|} \int_{0}^{w_{Q}} \lambda^{\delta} w(\{x \in Q: w(x)>\lambda\}) \frac{d \lambda}{\lambda}+\frac{\delta}{|Q|} \int_{w_{Q}}^{\infty} \lambda^{\delta} w(\{x \in Q: w(x)>\lambda\}) \frac{d \lambda}{\lambda} \\
& =I+I I .
\end{aligned}
$$

Observe that $I \leq\left(w_{Q}\right)^{\delta+1}$, where $w_{Q}=\frac{w(Q)}{|Q|}$.

To estimate $I I$ we make two claims. The first is the following observation: if we let

$$
E_{Q}=\left\{x \in Q: w(x) \leq \frac{1}{2[w]_{A_{2}}} w_{Q}\right\}
$$

then

$$
\left|E_{Q}\right| \leq \frac{1}{2}|Q|
$$

Indeed, by (Cauchy-Schwarz) we have for any $f \geq 0$,

$$
\left(\frac{1}{|Q|} \int_{Q} f(y) d y\right)^{2} w(Q) \leq[w]_{A_{2}} \int_{Q} f(y)^{2} w(y) d y
$$

and hence if $E \subset Q$, setting $f=\chi_{E}$,

$$
\left(\frac{|E|}{|Q|}\right)^{2} \leq[w]_{A_{2}} \frac{w(E)}{w(Q)}
$$

and in particular, by the definition of $E_{Q}$,

$$
\left(\frac{\left|E_{Q}\right|}{|Q|}\right)^{2} \leq[w]_{A_{2}} \frac{w\left(E_{Q}\right)}{w(Q)} \leq[w]_{A_{2}} \frac{w_{Q}}{w(Q)}\left|E_{Q}\right| \frac{1}{2[w]_{A_{2}}}=\frac{1}{2} \frac{\left|E_{Q}\right|}{|Q|}
$$

from which the claim follows. In particular, this implies that

$$
|Q| \leq 2\left|Q \backslash E_{Q}\right|=2\left|\left\{x \in Q: w(x)>\frac{1}{2[w]_{A_{2}}} w_{Q}\right\}\right| .
$$

The second claim is the following:

$$
w(\{x \in Q: w(x)>\lambda\}) \leq 2^{n+1} \lambda\left|\left\{x \in Q: w(x)>\frac{\lambda}{2[w]_{A_{p}}} w_{Q}\right\}\right|, \quad \lambda>w_{Q} .
$$

Indeed, since $\lambda>w_{Q}$, to prove this claim we consider the standard (local) CalderónZygmund decomposition of $w$ at level $\lambda$. Then there is a family of disjoint cubes $\left\{Q_{i}\right\}$ contained in $Q$ satisfying

$$
\lambda<w_{Q_{i}} \leq 2^{n} \lambda
$$

for each $i$. Now, observe that except for a null set we have

$$
\{x \in Q: w(x)>\lambda\} \subset\left\{x \in Q: M_{Q}^{d} w(x)>\lambda\right\}=\bigcup_{i} Q_{i},
$$


where $M_{Q}^{d}$ is the dyadic maximal operator restricted to a cube $Q$. This together with (5.3) yields

$$
\begin{gathered}
w(\{x \in Q: w(x)>\lambda\}) \leq \sum_{i} w\left(Q_{i}\right) \\
\leq 2^{n} \lambda \sum_{i}\left|Q_{i}\right| \leq 2^{n+1} \lambda \sum_{i}\left|\left\{x \in Q_{i}: w(x)>\frac{1}{2[w]_{A_{2}}} w_{Q_{i}}\right\}\right| \\
\leq 2^{n+1} \lambda\left|\left\{x \in Q: w(x)>\frac{1}{2[w]_{A_{2}}} \lambda\right\}\right|
\end{gathered}
$$

since $w_{Q_{i}}>\lambda$. This proves the second claim (5.4).

Now, combining

$$
\begin{aligned}
I I & =\frac{\delta}{|Q|} \int_{w_{Q}}^{\infty} \lambda^{\delta} w(\{x \in Q: w(x)>\lambda\}) \frac{d \lambda}{\lambda} \\
& \leq \frac{2^{n+1} \delta}{|Q|} \int_{w_{Q}}^{\infty} \lambda^{\delta+1}\left|\left\{x \in Q: w(x)>\frac{1}{2[w]_{A_{2}}} \lambda\right\}\right| \frac{d \lambda}{\lambda} \\
& \leq\left(2[w]_{A_{2}}\right)^{1+\delta} 2^{n+1} \delta \frac{1}{|Q|} \int_{\frac{w_{Q}}{2[w]_{A_{2}}}}^{\infty} \lambda^{\delta+1}|\{x \in Q: w(x)>\lambda\}| \frac{d \lambda}{\lambda} \\
& \leq\left(2[w]_{A_{2}}\right)^{1+\delta} 2^{n+1} \frac{\delta}{1+\delta} \frac{1}{|Q|} \int_{Q} w^{1+\delta} d x .
\end{aligned}
$$

Setting here $\delta=\frac{1}{2^{5+n}[w]_{A_{2}}}$, we obtain using that $t^{1 / t} \leq 2, t \geq 1$,

$$
I I \leq \frac{1}{2} \frac{1}{|Q|} \int_{Q} w^{\delta+1} d x
$$

and finally

$$
\frac{1}{|Q|} \int_{Q} w^{\delta+1} d x \leq 2\left(w_{Q}\right)^{\delta+1}
$$

which proves (5.1).

\section{REFERENCES}

[1] J. Alvarez, R. Bagby, D. Kurtz, and C. Perez, Weighted estimates for commutators of linear operators, Studia Math. 104 (2) (1994) 195-209. MR1211818 (94k:47044)

[2] K. Astala, T. Iwaniec, and E. Saksman, Beltrami operators in the plane, Duke Mathematical Journal 107 (2001), no. 1, 27-56. MR.1815249 (2001m:30021)

[3] O. Beznosova, Linear bound for dyadic paraproduct on weighted Lebesgue space $L^{2}(w)$, J. Funct. Anal. 255 (2008), no. 4, 994-1007. MR2433959 (2009e:42040)

[4] S. M. Buckley, Estimates for operator norms on weighted spaces and reverse Jensen inequalities, Trans. Amer. Math. Soc., 340 (1993), no. 1, 253-272. MR1124164 (94a:42011)

[5] D. Chung, Sharp estimates for the commutators of the Hilbert, Riesz and Beurling transforms on weighted Lebesgue spaces, Indiana U. Math. J., to appear, Preprint (2010) available at http://arxiv.org/abs/1001.0755

[6] R. Coifman and C. Fefferman, Weighted norm inequalities for maximal functions and singular integrals, Studia Math. 51 (1974),241-250. MR0358205 (50:10670)

[7] R. Coifman, R. Rochberg, and G. Weiss, Factorization theorems for Hardy spaces in several variables, Ann. of Math. (2) 103 (1976), 611-635. MR0412721(54:843)

[8] D. Cruz-Uribe, J.M. Martell and C. Pérez, Sharp weighted estimates for approximating dyadic operators, Electronic Research Announcements in the Mathematical Sciences, 17 (2010), 12 19. MR2628851 
[9] D. Cruz-Uribe, J. Martell, and C. Pérez, Sharp weighted estimates for classical operators, to appear Adv. Math., available at http://www.arxiv.org/abs/1001.4254

[10] D. Cruz-Uribe, J. Martell, and C. Pérez, Weights, Extrapolation and the Theory of Rubio de Francia.(Operator Theory: Advances and Applications) Springer Basel; 1st Edition. edition (April 28, 2011).

[11] D. Cruz-Uribe and K. Moen, Sharp norm inequalities for commutators of classical operators, to appear Publ. Math., available at http://www.arxiv.org/abs/1008.0381

[12] O. Dragičević, L. Grafakos, C. Pereyra, and S. Petermichl, Extrapolation and sharp norm estimates for classical operators on weighted Lebegue spaces, Publ. Math. 49 (2005), no. 1, 73-91. MR2140200 (2006d:42019)

[13] O. Dragičević and A. Volberg, Sharp estimate of the Ahlfors-Beurling operator via averaging Martingale transforms, Michigan Math. J. 51 (2003) no. 2, 415-435. MR1992955 (2004c:42030)

[14] J. García-Cuerva and J. L. Rubio de Francia, Weighted Norm Inequalities and Related Topics, North-Holland, Amsterdam, 1985. MR807149 (87d:42023)

[15] S. Hukovic, S. Treil, and A. Volberg, The Bellman functions and the sharp weighted inequalities for square function, Operator Theory: Advances and Applications, the volume in memory of S. A. Vinogradov 113, Birkhäuser Verlag (2000). MR1771755 (2001j:42012)

[16] T. Hytönen, The sharp weighted bound for general Calderón-Zygmund operators, to appear Annals of Math., available at http://arxiv.org/abs/1007.4330

[17] T. Hytönen, C. Pérez, S. Treil, and A. Volberg, Sharp weighted estimates of the dyadic shifts and $A_{2}$ conjecture, Preprint (2010) available at http://arxiv.org.abs/1010.0755

[18] F. John and L. Nirenberg, On functions of bounded mean oscillation, Comm. Pure Appl. Math. 14 (1961) 415-426. MR0131498 (24:A1348)

[19] J. L. Journé, Calderón-Zygmund operators, pseudo-differential operators and the Cauchy integral of Calderón, Lect. Notes Math. 994, Springer Verlag, (1983). MR706075 (85i:42021)

[20] M. Lacey, S. Petermichl, and M. C. Reguera, Sharp $A_{2}$ inequality for Haar shift operators, Math. Ann. 348 (2010), no. 1, 127-141. MR2657437

[21] A. K. Lerner, An elementary approach to several results on the Hardy-Littlewood maximal operator, Proc. Amer. Math. Soc. 136 (2008) no. 8, 2829-2833. MR2399047(2009c:42047)

[22] A. K. Lerner, Sharp weighted norm inequalities for Littlewood-Paley operators and singular integrals, Adv. Math. 226 (2011) no. 5, 3912-3926. MR2770437

[23] A. Lerner, S. Ombrosi and C. Pérez, Sharp $A_{1}$ bounds for Calderón-Zygmund operators and the relationship with a problem of Muckenhoupt and Wheeden, International Mathematics Research Notices, 2008, no. 6, Art. ID rnm161, 11 pp. 42B20.

[24] A. Lerner, C. Pérez and S. Ombrosi, $A_{1}$ bounds for Calderón-Zygmund operators related to a problem of Muckenhoupt and Wheeden, Math. Res. Lett. 16 (2009), 149-156. MR2480568 (2010a:42052)

[25] C. Ortiz, Quadratic $A_{1}$ bounds for commutators of singular integrals with BMO functions, Indiana U. Math. J., to appear.

[26] C. Pérez, Endpoint Estimates for Commutators of Singular Integral Operators, Journal of Functional Analysis, (1) 128 (1995), 163-185. MR1317714 (95j:42011)

[27] C. Pérez, Sharp estimates for commutators of singular integrals via iterations of the Hardy-Littlewood maximal function, J. Fourier Anal. Appl. 3 (1997), 743-756. MR 1481632 (99f:42042)

[28] C. Pérez, A course on Singular Integrals and weights, to appear in Advanced Courses in Mathematics, CRM Barcelona, Birkhäuser editors.

[29] C. Pérez, S. Treil, and A. Volberg, On $A_{2}$ conjecture and corona decomposition of weights. Preprint 2010, available at http://arxiv.org/abs/1006.2630.

[30] S. Petermichl, The sharp bound for the Hilbert transform on weighted Lebesgue spaces in terms of the classical $A_{p}$ characteristic, Amer. J. of Math. 129 (2007) 1355-1375. MR2354322 (2008k:42066)

[31] S. Petermichl, The sharp weighted bound for the Riesz transforms, Proc. Amer. Math. Soc. 136 (2008) 1237-1249. MR 2367098 (2009c:42034)

[32] S. Petermichl and A. Volberg, Heating of the Ahlfors-Beurling operator: Weakly quasiregular maps on the plane are quasiregular, Duke Math. J. 112 (2002) 281-305. MR.1894362 (2003d:42025) 
[33] A. Torchinsky, Real-Variable Methods in Harmonic Analysis, Academic Press, New York, (1986). MR869816 (88e:42001)

[34] J. Wittwer, A sharp estimate on the norm of the martingale transform, Math. Res. Lett. 7 (2000) 1-12. MR 1748283(2001e:42022)

Department of Mathematics and Statistics MSC01 1115, University of New Mexico, Albuquerque, New MeXico 87131-0001

E-mail address: midiking@math.unm.edu

Department of Mathematics and Statistics, MSC01 1115, University of New Mexico, Albuquerque, New MeXico 87131-0001

E-mail address: crisp@math.unm.edu

Departamento de Análisis Matemático, Facultad de Matemáticas, Universidad De Sevilla, 41080 Sevilla, Spain

E-mail address: carlosperez@us.es 